\title{
Cubic Polynomial for the Series of Consecutive Cubes under Alternating Signs
}

\author{
Leomarich F. Casinillo ${ }^{1 *}$ and Crisanto L. $\mathrm{Abas}^{2}$ \\ 1,2 Department of Mathematics, Visayas State University, Visca, Baybay City, Leyte, Philippines \\ ${ }^{*}$ Corresponding e-mail: leomarichcasinillo02011990@gmail.com
}

\begin{abstract}
This paper aims to develop an elegant formula for the series of consecutive cubes of natural numbers under alternating signs. In addition, this paper investigates the formula under odd and even number of terms and discuss some important findings.

Keywords: consecutive cubes; alternating signs; odd and even terms.

2010 Mathematics Subject Classification: 11B13, 11B50, 97I30
\end{abstract}

\begin{abstract}
Abstrak
Paper ini bertujuan membangun formula yang elegan untuk deret berganti tanda bilangan-bilangan kubik berurutan. Paper ini juga menyelidiki formula untuk banyak suku ganjil dan untuk banyak suku genap, dan mendiskusikan beberapa temuan penting.

Kata kunci: bilangan kubik berurutan; berganti tanda; suku ganjil dan suku genap.
\end{abstract}

\section{INTRODUCTION}

Mathematicians have worked to find a constructive formula for the following equation (see [1] [2] [3] [4] [5]):

$$
\lambda^{p}+(\lambda+1)^{p}+\cdots+(\lambda+t-1)^{p}=\sum_{j=\lambda}^{\lambda+t-1} j^{p}
$$

where $\lambda$ and $t$ are any positive integers. In this paper, our focus is the sum of the first $t$ terms of consecutive cubes denoted by $S_{t}^{o}(\lambda, x)$ and $S_{t}^{e}(\lambda, x)$, where the superscript $O$ and $e$ stands for odd and even terms, respectively. That is,

$$
S_{t}^{o}(\lambda, x)=\lambda^{3}+(\lambda+1)^{3}+\cdots+(\lambda+2 x-2)^{3}=\sum_{j=\lambda}^{\lambda+2 x-2} j^{3},
$$

where $\lambda, x$ are positive integers, $t=2 x-1$, and

$$
S_{t}^{e}(\lambda, x)=\lambda^{3}+(\lambda+1)^{3}+\cdots+(\lambda+2 x-1)^{3}=\sum_{j=\lambda}^{\lambda+2 x-1} j^{3},
$$

where $\lambda, x$ are positive integers, and $t=2 x$. We have that

$$
S_{t}(1, x)=1^{3}+2^{3}+\cdots+t^{3}=\sum_{j=1}^{t} j^{3}=\frac{t^{3}(t+1)^{2}}{4},
$$

where $x$ is a positive integer and $t=f(x)$. The equation (4) can be proven by mathematical induction. Forth, we extend equation by Casinillo and Mamolo [6], which dealing with an alternating consecutive square of positive integers. Hence, the goal of this paper is to construct an equation of an alternating

Submitted March 19th, 2021, Revised August 23 ${ }^{\text {rd }}$, 2021, Accepted for publication August 24th, 2021.

This is an open access article under CC-BY-SA license (https://creativecommons.org/licence/by-sa/4.0/) 
sum of the first $t$ terms of consecutive cubes denoted by $A_{t}^{o}(\lambda, x)$ and $A_{t}^{e}(\lambda, x)$, where the superscript $o$ and $e$ stands for odd and even terms, respectively. If $\lambda$ is even, we have

$$
A_{t}^{o}(\lambda, x)=\lambda^{3}-(\lambda+1)^{3}+\cdots+(\lambda+2 x-2)^{3}=\sum_{j=\lambda}^{\lambda+2 x-2}(-1)^{j} j^{3},
$$

where $x$ is a positive integer and $t=2 x-1$. And if $\lambda$ is odd, we also have

$$
A_{t}^{o}(\lambda, x)=\lambda^{3}-(\lambda+1)^{3}+\cdots+(\lambda+2 x-2)^{3}=\sum_{j=\lambda}^{\lambda+2 x-2}(-1)^{j+1} j^{3},
$$

where $x$ is a positive integer and $t=2 x-1$. And for alternating even terms, we have

$$
A_{t}^{e}(\lambda, x)=\lambda^{3}-(\lambda+1)^{3}+\cdots-(\lambda+2 x-1)^{3}=\sum_{j=\lambda}^{\lambda+2 x-1}(-1)^{j} j^{3},
$$

for $\lambda$ even and $x$ is a positive integer and $t=2 x$. Finally, if $\lambda$ is odd, we have

$$
A_{t}^{e}(\lambda, x)=\lambda^{3}-(\lambda+1)^{3}+\cdots-(\lambda+2 x-1)^{3}=\sum_{j=\lambda}^{\lambda+2 x-1}(-1)^{j+1} j^{3},
$$

where $x$ is a positive integer and $t=2 x$. In this paper, we developed a cubic polynomial formula that computes the sum of consecutive cubes under alternating signs. Furthermore, we had also investigated the formula under even and odd terms.

\section{RESULTS}

Our first theorem is quick from equation (5) and (6) above when $t$ is odd. The theorem shows the cubic polynomial for the sums of consecutive cubes under alternating signs with odd terms.

Theorem 1. Let $\lambda$ and $x$ be positive integers. If $x=\frac{t+1}{2}$ where $t \equiv 1(\bmod 2)$, then

$$
A_{t}^{o}(\lambda, x)=4 x^{3}+(6 \lambda-9) x^{2}+\left(3 \lambda^{2}-9 \lambda+6\right) x+(\lambda-1)^{3}>0 .
$$

Proof. Let $x$ be a positive integer. Since $A_{t}^{o}(\lambda, x)$ is a cubic polynomial in nature, then we suppose that $A_{t}^{o}(\lambda, x)=a_{3} x^{3}+a_{2} x^{2}+a_{1} x+a_{0}$ where $a_{i}=f_{i}(\lambda), i=1,2,3,4$ are positive integers and functions of positive integer $\lambda$. To solve for coefficients $a_{i}$, we let $x=1,2,3,4$. Then, we obtain the following system of equations

$$
\left\{\begin{array}{c}
a_{3}+a_{2}+a_{1}+a_{0}=\lambda^{3} \\
8 a_{3}+4 a_{2}+2 a_{1}+a_{0}=\sum_{j=0}^{2}(-1)^{j}(\lambda+j)^{3} \\
27 a_{3}+9 a_{2}+3 a_{1}+a_{0}=\sum_{j=0}^{4}(-1)^{j}(\lambda+j)^{3} \\
64 a_{3}+16 a_{2}+4 a_{1}+a_{0}=\sum_{j=0}^{6}(-1)^{j}(\lambda+j)^{3} .
\end{array}\right.
$$

By Gaussian Elimination, expression (10) rewrite by following

$$
\left\{\begin{aligned}
a_{3}+a_{2}+a_{1}+a_{0} & =\lambda^{3} \\
7 a_{3}+3 a_{2}+a_{1} & =3 \lambda^{2}+9 \lambda+7 \\
42 a_{3}+6 a_{2} & =36 \lambda+114 \\
a_{3} & =4
\end{aligned}\right.
$$

Under backward substitution in (11), we have the following values for $a_{i}, i=0,1,2,3$.

$$
\left\{\begin{array}{l}
a_{0}=(\lambda-1)^{3} \\
a_{1}=3 \lambda^{2}-9 \lambda+6 \\
a_{2}=6 \lambda-9 \\
a_{3}=4
\end{array}\right.
$$

87 | InPrime: Indonesian Journal of Pure and Applied Mathematics 
Moreover in (12), for $\lambda \geq 2, a_{i}>0$ for all $i$ and for $\lambda=1, a_{3}+a_{2}+a_{1}+a_{0}>0$. Thus, $A_{t}^{o}(\lambda, x)>0$ for all positive integer $x$. Next, we verify that equation (9) holds for all positive integer. Without loss of generality (WLOG), for $\lambda \equiv 0(\bmod 2), A(x)$ be

$$
A_{t}^{o}(\lambda, x)=\sum_{j=\lambda}^{\lambda+2 x-2}(-1)^{j} j^{3}=4 x^{3}+(6 \lambda-9) x^{2}+\left(3 \lambda^{2}-9 \lambda+6\right) x+(\lambda-1)^{3},
$$

where $t=2 x-1$ and $\lambda$ be any positive integer. For $x=1$, the statement $A(x)$ is true, that is

$$
\lambda^{3}=4(1)^{3}+(6 \lambda-9)(1)^{2}+\left(3 \lambda^{2}-9 \lambda+6\right)(1)+(\lambda-1)^{3}=\lambda^{3} .
$$

Note that, the induction hypothesis is given by the statement $A(x)$. Now, we want to show that equation (13) holds for $x+1$, that is, the statement $A(x+1)$. So, we have

$$
A_{t+2}^{o}(\lambda, x+1)=\sum_{j=\lambda}^{\lambda+2 x-2}(-1)^{j} j^{3}-(\lambda+2 x-1)^{3}+(\lambda+2 x)^{3} .
$$

From our assumption, we obtain

$A_{t+2}^{o}(\lambda, x+1)=4 x^{3}+(6 \lambda-9) x^{2}+\left(3 \lambda^{2}-9 \lambda+6\right) x+(\lambda-1)^{3}-(\lambda+2 x-1)^{3}+(\lambda+2 x)^{3}$.

By directly expanding, simplifying and factoring terms in (15), we have

$$
A_{t+2}^{o}(\lambda, x+1)=4(x+1)^{3}+(6 \lambda-9)(x+1)^{2}+\left(3 \lambda^{2}-9 \lambda+6\right)(x+1)+(\lambda-1)^{3} .
$$

Thus, $A(x)$ is true for all positive integer $x$ and hypothesis follows. This completes the proof.

\section{Illustration}

The following table shows the alternating sum for the first 10 values of $\lambda$.

Table 1. Alternating sum for the first $10 \lambda$ 's

\begin{tabular}{ccc}
\hline $\boldsymbol{\lambda}$ & $\boldsymbol{A}_{\boldsymbol{t}}^{o}(\boldsymbol{\lambda}, \boldsymbol{x})$ & Expanded Form $(\boldsymbol{t}=\mathbf{2} \boldsymbol{x}-\mathbf{1})$ \\
\hline 1 & $A_{t}^{o}(1, x)=4 x^{3}-3 x^{2}$ & $1^{3}-2^{3}+\cdots+t^{3}$ \\
\hline 2 & $A_{t}^{o}(2, x)=4 x^{3}+3 x^{2}+1$ & $2^{3}-3^{3}+\cdots+t^{3}$ \\
\hline 3 & $A_{t}^{o}(3, x)=4 x^{3}+9 x^{2}+6 x+8$ & $3^{2}-4^{3}+\cdots+t^{3}$ \\
\hline 4 & $A_{t}^{o}(4, x)=4 x^{3}+15 x^{2}+18 x+27$ & $4^{2}-5^{3}+\cdots+t^{3}$ \\
\hline 5 & $A_{t}^{o}(5, x)=4 x^{3}+21 x^{2}+36 x+64$ & $5^{3}-6^{3}+\cdots+t^{3}$ \\
\hline 6 & $A_{t}^{o}(6, x)=4 x^{3}+27 x^{2}+60 x+125$ & $6^{3}-7^{3}+\cdots+t^{3}$ \\
\hline 7 & $A_{t}^{o}(7, x)=4 x^{3}+33 x^{2}+90 x+216$ & $7^{3}-8^{3}+\cdots+t^{3}$ \\
\hline 8 & $A_{t}^{o}(3, x)=4 x^{3}+39 x^{2}+126 x+343$ & $8^{3}-9^{3}+\cdots+t^{3}$ \\
\hline 9 & $A_{t}^{o}(9, x)=4 x^{3}+45 x^{2}+168 x+512$ & $9^{3}-10^{3}+\cdots+t^{3}$ \\
\hline 10 & $A_{t}^{o}(10, x)=4 x^{3}+51 x^{2}+216 x+729$ & $10^{3}-11^{3}+\cdots+t^{3}$ \\
\hline
\end{tabular}

The following corollary is a consequence from Theorem 1. This result shows the cubic polynomial formula for the series of consecutive cubes for at most 7 terms, that is, $t \leq 7$ where $t$ is odd. 
Corollary 1. Let $\lambda$ and $x$ be positive integers. If $x=\frac{t+1}{2} \leq 4$ where $t \equiv 1(\bmod 2)$, then

$$
\begin{aligned}
S_{t}^{o}(\lambda, x)= & (8 \lambda+28) x^{3}+\left(6 \lambda^{2}-18 \lambda-127\right) x^{2}+\left(2 \lambda^{3}-9 \lambda^{2}+13 \lambda+194\right) x+ \\
& \left(-\lambda^{3}+3 \lambda^{2}-3 \lambda-95\right) .
\end{aligned}
$$

The next theorem shows the cubic polynomial for the sums of consecutive cubes with even terms.

Theorem 2. Let $\lambda$ and $x$ be positive integers. If $x=\frac{t}{2}$ where $t \equiv 0(\bmod 2)$, then

$$
A_{t}^{o}(\lambda, x)=-4 x^{3}+(-6 \lambda+3) x^{2}+\left(-3 \lambda^{2}+3 \lambda\right) x<0
$$

Proof. Let $x$ be a positive integer. Since $A_{t}^{o}(\lambda, x)$ is a cubic polynomial in nature, then we suppose that $A_{t}^{o}(\lambda, x)=a_{3} x^{3}+a_{2} x^{2}+a_{1} x+a_{0}$ where $a_{i}=f_{i}(\lambda), i=0,1,2,3$ are positive integers and functions of positive integer $\lambda$. To solve for coefficients $a_{i}$, we let $x=1,2,3,4$. Then, we obtain the following systems of equations

$$
\left\{\begin{aligned}
a_{3}+a_{2}+a_{1}+a_{0} & =\sum_{j=0}^{1}(-1)^{j}(\lambda+j)^{3} \\
8 a_{3}+4 a_{2}+2 a_{1}+a_{0} & =\sum_{j=0}^{3}(-1)^{j}(\lambda+j)^{3} \\
27 a_{3}+9 a_{2}+3 a_{1}+a_{0} & =\sum_{j=0}^{5}(-1)^{j}(\lambda+j)^{3} \\
64 a_{3}+16 a_{2}+4 a_{1}+a_{0} & =\sum_{j=0}^{7}(-1)^{j}(\lambda+j)^{3}
\end{aligned}\right.
$$

By Gaussian Elimination, we can rewrite (18) as follows

$$
\left\{\begin{aligned}
a_{3}+a_{2}+a_{1}+a_{0} & =-3 \lambda^{2}-3 \lambda-1 \\
63 a_{3}+15 a_{2}+3 a_{1} & =-9 \lambda^{2}-81 \lambda-207 \\
42 a_{3}+6 a_{2} & =-36 \lambda-150 \\
a_{3} & =-4
\end{aligned}\right.
$$

Then, under back substitution in (19), we obtain the following values for $a_{i}, i=0,1,2,3$,

$$
\left\{\begin{array}{l}
a_{0}=0 \\
a_{1}=-3 \lambda^{2}+3 \lambda \\
a_{2}=-6 \lambda+3 \\
a_{3}=-4
\end{array}\right.
$$

Moreover in (20), since $\lambda>0, a_{i} \leq 0$ for all $i=0,1,2,3$, we have $A_{t}^{o}(\lambda, x)<0$ for all positive integer $x$. Next, we verify that equation (17) holds for all positive integer $x$. WLOG, let $\lambda \equiv$ $0(\bmod 2)$. Hence, we let $B(x)$ be

$$
A_{t}^{e}(\lambda, x)=\sum_{j=\lambda}^{\lambda+2 x-1}(-1)^{j} j^{3}=-4 x^{3}+(-6 \lambda+3) x^{2}+\left(-3 \lambda^{2}+3 \lambda\right) x,
$$

where $t=2 x$ and $\lambda$ any positive integer. For $x=1, B(x)$ is true, that is

$$
\begin{aligned}
\lambda^{3}-(\lambda+1)^{3} & =-4(1)^{3}+(-6 \lambda+3)(1)^{2}+\left(-3 \lambda^{2}+3 \lambda\right)(1) \\
-3 \lambda^{2}-3 \lambda-1 & =-3 \lambda^{2}-3 \lambda-1
\end{aligned}
$$

Our induction hypothesis is given by the statement $B(x)$. So, we show that equation (21) is hold for $x+1$, that is, the statement $B(x+1)$. So, we have 


$$
A_{t+2}^{e}(\lambda, x+1)=\sum_{j=\lambda}^{\lambda+2 x-1}(-1)^{j} j^{3}+(\lambda+2 x)^{3}-(\lambda+2 x+1)^{3} .
$$

Hence, from our assumption, it follows that

$$
A_{t+2}^{e}(\lambda, x+1)=-4 x^{3}+(-6 \lambda+3) x^{2}+\left(-3 \lambda^{2}+3 \lambda\right) x+(\lambda+2 x)^{3}-(\lambda+2 x+1)^{3} .
$$

By directly expanding, simplifying and factoring terms in equation (23), it implies that

$$
A_{t+2}^{e}(\lambda, x+1)=-4(x+1)^{3}+(-6 \lambda+3)(x+1)^{2}+\left(-3 \lambda^{2}+3 \lambda\right)(x+1) .
$$

Thus, $B(x)$ is true for all positive integer $x$ and hypothesis follows. This completes the proof.

\section{Illustration}

The following table shows the sum for the first 10 values of $\lambda$ for even terms.

Table 2. Alternating sum for the first $10 \lambda$ 's

\begin{tabular}{ccc}
\hline$\lambda$ & $\boldsymbol{A}_{\boldsymbol{t}}^{e}(\boldsymbol{\lambda}, \boldsymbol{x})$ & Expanded Form $(\boldsymbol{t}=\mathbf{2 x})$ \\
\hline 1 & $A_{t}^{e}(1, x)=-4 x^{3}-3 x^{2}$ & $1^{3}-2^{3}+\cdots-t^{3}$ \\
\hline 2 & $A_{t}^{e}(2, x)=-4 x^{3}-9 x^{2}-6 x$ & $2^{3}-3^{3}+\cdots-t^{3}$ \\
\hline 3 & $A_{t}^{e}(3, x)=-4 x^{3}-15 x^{2}-18 x$ & $3^{3}-4^{3}+\cdots-t^{3}$ \\
\hline 4 & $A_{t}^{e}(4, x)=-4 x^{3}-21 x^{2}-36 x$ & $4^{3}-5^{3}+\cdots-t^{3}$ \\
\hline 5 & $A_{t}^{e}(5, x)=-4 x^{3}-27 x^{2}-60 x$ & $5^{3}-6^{3}+\cdots-t^{3}$ \\
\hline 6 & $A_{t}^{e}(6, x)=-4 x^{3}-33 x^{2}-90 x$ & $6^{3}-7^{3}+\cdots-t^{3}$ \\
\hline 7 & $A_{t}^{e}(7, x)=-4 x^{3}-39 x^{2}-126 x$ & $7^{3}-8^{3}+\cdots-t^{3}$ \\
\hline 8 & $A_{t}^{e}(8, x)=-4 x^{3}-45 x^{2}-168 x$ & $8^{3}-9^{3}+\cdots-t^{3}$ \\
\hline 9 & $A_{t}^{e}(9, x)=-4 x^{3}-51 x^{2}-216 x$ & $9^{3}-10^{3}+\cdots-t^{3}$ \\
\hline 10 & $A_{t}^{e}(10, x)=-4 x^{3}-57 x^{2}-270 x$ & $10^{3}-11^{3}+\cdots-t^{3}$ \\
\hline
\end{tabular}

Corollary below is an immediate from Theorem 2 showing the cubic polynomial formula for the series of consecutive cubes with at most 8 terms, that is, $8 \geq t \equiv 0(\bmod 2)$.

Corollary 2. Let $\lambda$ and $x$ be positive integers. If $x=\frac{t}{2} \leq 4$ where $t \equiv 0(\bmod 2)$, then

$$
S_{t}^{e}(\lambda, x)=(8 \lambda+36) x^{3}+\left(6 \lambda^{2}-6 \lambda-139\right) x^{2}+\left(2 \lambda^{3}-3 \lambda^{2}+\lambda+200\right) x-96 .
$$

\section{CONCLUSION}

This paper developed new formula for the series of natural number $t$ consecutive cubes under alternating signs. The formula is constructed in a cubic polynomial form which is a function of two positive integers. It is concluded that series with odd term and even term have positive and negative values, respectively, i.e., $A_{t}^{o}(\lambda, x)>0$ and $A_{t}^{e}(\lambda, x)<0$. Both cubic polynomial formula for $A_{t}^{o}(\lambda, x)$ and $A_{t}^{e}(\lambda, x)$ is proven by mathematical induction. Furthermore, we also developed a cubic polynomial 
formula for sum of cubes, that is $S_{t}^{o}(\lambda, x)$ and $S_{t}^{e}(\lambda, x)$, in which it is only valid for at most 7 and 8 terms in the series, respectively.

\section{Acknowledgements}

The authors would like to acknowledge the referees for the rigorous review and comments to improve this paper.

\section{REFERENCES}

[1] G. F. C. de Bruyn and J. M. de Villiers, "Formulas for $1^{\wedge} \mathrm{p}+2^{\wedge} \mathrm{p}+\ldots+\mathrm{n} \wedge \mathrm{p} ., "$ The Fibonacci Quarterly, vol. 32, no. 3, pp. 271-276, 1994.

[2] F. T. Howard, "Sums of powers of integers via generating functions," Fibonacci Quarterly, vol. 34, no. Retrieved from https://www.fq.math.ca/Scanned/34-3/howard.pdf, pp. 244-256, 1996.

[3] D. Kalman, "Sums of Powers by Matrix Methods," The Fibonacci Quarterly, vol. 28, no. 1, pp. 6071, 1990.

[4] M. Merca, "An alternative to Faulhaber's formula," The American Mathematical Monthly, vol. 122, no. 6. DOI: 10.4169/amer.math.monthly.122.6.599, pp. 599-601, 2014.

[5] B. Turner, "Sums of Powers of Integers via the Binomial Theorem," Mathematics Magazine, vol. 53, pp. 92-96, 1980.

[6] L. F. Casinillo and L. A. Mamolo, "Alternative Formula for the Series of Consecutive m-Squares under Alternating Signs," InPrime: Indonesian Journal of Pure and Applied Mathematics, vol. 2, no. 2, pp. 91-96, 2020. 